\title{
THE ARF INVARIANT FOR KNOT TYPES
}

\section{KUNIO MURASUGI}

The purpose of this paper is to prove Theorem 1 below which gives a simple relation between the Arf invariant $\phi(k)$ and the Minkowski unit $C_{p}(k), p=2$, of a tame knot $k$ in 3-space.

TheOREM 1. $C_{2}(k)=(-1)^{\phi(k)}$.

The cobordism invariance of $\phi(k)$ which is proved by Robertello [3] follows from this theorem and Corollary 3.5 in [2]. Further, if we denote by $\Delta(t)$ the Alexander polynomial of $k$, then a simple calculation leads to the following

Theorem 2. $\phi(k)=0$ iff $\Delta(-1) \equiv \pm 1(\bmod 8)$.

1. Seifert matrix. Let $k$ be an oriented tame knot in 3-space and let $S$ be a Seifert surface of $k . S$ is a 2 -cell with $2 h$ bands $B_{1}, \cdots, B_{2 h}$, where $h$ is the genus of $S$. Let $V_{2 h}=\left(v_{i j}\right)$ be the Seifert matrix associated to $S$. $V_{2 h}$ is an integral $2 h \times 2 h$ matrix and the $(i, j)$ entry of the symmetric matrix $M=V_{2 h}+V_{2 h}^{\prime}$ is odd iff $(i, j)=(2 r-1,2 r)$ or $(2 r, 2 r-1)$, and hence $\operatorname{det} M$ is odd.

Let $V_{m}(m \leqq 2 h)$ be the principal minor consisting of the first $m$ rows and columns of $V_{2 h}$. Then $V_{2 l}$ can be considered as the Seifert matrix associated to the surface $S^{\prime}$ obtained from $S$ by removing $2 h-2 l$ bands $B_{2 l+1}, \cdots, B_{2 h}$. Let $D_{m}=\operatorname{det}\left(V_{m}+V_{m}^{\prime 3}\right)$.

Lemma 1 . Let $1 \leqq n \leqq h$ and $D_{0}=1$. Then $D_{2 n-2} D_{2 n} \equiv-1$ or $3(\bmod 8)$ according as $v_{2 n-1,2 n-1} v_{2 n, 2 n}$ is even or odd. Moreover, $D_{2 n-1}$ is even, but if $v_{2 n-1,2 n-1}$ is odd then $D_{2 n-1} \equiv 2(\bmod 4)$.

Proof. Let $M=V_{2 n}+V_{2 n}^{\prime}$. We know ${ }^{1}$ that

$$
D_{2 n-2} D_{2 n}=D_{2 n-1} \operatorname{det} \tilde{M}(2 n-1)-\left\{\operatorname{det} \tilde{M}\left(\begin{array}{c}
2 n-1 \\
2 n
\end{array}\right)\right\}^{2} .
$$

(For a proof, for example, see [1, p. 7].) Since

$$
\operatorname{det} \tilde{M}\left(\begin{array}{c}
2 n-1 \\
2 n
\end{array}\right)
$$

is odd,

Received by the editors March 8, 1968.

$1 \widetilde{M}\left(\begin{array}{l}p \\ )\end{array}\right)$ denotes the matrix obtained from $M$ by deleting the $p$ th row and $q$ th column, and $\tilde{M}(p)=\tilde{M}\left(\begin{array}{l}p \\ p\end{array}\right)$. 


$$
\left\{\operatorname{det} \tilde{M}\left(\begin{array}{c}
2 n-1 \\
2 n
\end{array}\right)\right\}^{2} \equiv 1(\bmod 8) .
$$

Hence, $\quad D_{2 n-2} D_{2 n} \equiv D_{2 n-1} \quad \operatorname{det} \tilde{M}(2 n-1)-1 \quad(\bmod 8)$. Further, $\operatorname{det} \tilde{M}(2 n-1) \equiv 2 v_{2 n, 2 n} D_{2 n-2}(\bmod 4), D_{2 n-1} \equiv 2 v_{2 n-1,2 n-1} D_{2 n-2}(\bmod 4)$ and $D_{2 n-2}$ is odd. Therefore, $\operatorname{det} \tilde{M}(2 n-1) D_{2 n-1} \equiv 4 v_{2 n-1,2 n-1} v_{2 n, 2 n}$ $(\bmod 8)$ and $D_{2 n-1} \equiv 2 v_{2 n-1,2 n-1}(\bmod 4)$. This proves Lemma 1.

Since $D_{2 n-2} D_{2 n} \equiv-1(\bmod 4)$ by Lemma 1 , it follows

LEMMA 2. $D_{2 n} \equiv(-1)^{n}(\bmod 4)$ for $0 \leqq n \leqq h$.

Let $(a, b)_{2}$ denote the Hilbert symbol. Then Lemma 2 implies

LEMMA 3. $\left(-1, D_{2 n}\right)_{2}=(-1)^{n}$.

Further, we can prove

LeMMA 4. $\left(D_{2 n-1},-D_{2 n-2} D_{2 n}\right)_{2}=1$ or -1 according as $v_{2 n-1,2 n-1} v_{2 n, 2 n}$ is even or odd.

Proof. Let us write $D_{2 n-1}=2^{m} t$, where $m$ is a nonnegative integer, $t$ is odd and let $q=D_{2 n-2} D_{2 n}$. Then $\left(D_{2 n-1},-q\right)_{2}=(2,-q)_{2}^{m}(t,-q)_{2}$. If $v_{2 n-1,2 n-1} v_{2 n, 2 n}$ is even, then $q \equiv-1(\bmod 8)$ by Lemma 1 . Hence $(2,-q)_{2}=1$ and $(t,-q)_{2}=1$. Thus $\left(D_{2 n-1},-q\right)_{2}=1$. If $v_{2 n-1,2 n-1} v_{2 n, 2 n}$ is odd, then $D_{2 n-1}$ is not divisible by 4 , i.e. $m=1$, and $q \equiv 3(\bmod 8)$. Therefore, $\left(D_{2 n-1},-q\right)_{2}=(2,-q)_{2}(t,-q)_{2}=-1$.

2. Proof of Theorem 1. Given a Seifert matrix $V_{2 h}$ of a knot $k$, we can define the Arf invariant $\phi(k)[3]$ and the Minkowski unit $C_{2}(k)$ [2] as follows.

$$
\phi(k) \equiv \sum_{i=1}^{h} v_{2 i-1,2 i-1} v_{2 i, 2 i}(\bmod 2)
$$

and

$$
C_{2}(k)=(-1)^{\beta}\left(-1,-D_{2 h}\right)_{2} \prod_{i=1}^{2 h-1}\left(D_{i},-D_{i+1}\right)_{2},
$$

where $\beta=[h / 2]+(1+h)\left(D_{2 h}+1\right) / 2$.

Since $D_{2 h} \equiv(-1)^{h}(\bmod 4)$ by Lemma 2 , we see that $\beta \equiv[h / 2]+h$ $+1(\bmod 2)$.

Now Lemma 2 shows that $D_{2 n}$ is not zero, while $D_{2 n-1}$ may be zero. If $D_{2 n-1}$ is zero, $\left(D_{2 n-2},-D_{2 n-1}\right)_{2}$ and $\left(D_{2 n-1},-D_{2 n}\right)_{2}$ are interpreted to be $\left(D_{2 n-2},-1\right)_{2}$ and $\left(1,-D_{2 n}\right)_{2}$, respectively.

Now the proof of Theorem 1 will proceed by induction on $h$, the genus of $S$. 
For $h=0$, the theorem is obvious. Suppose $h=1$. Since $\beta=1$ and $\left(-1,-D_{2}\right)_{2}=1$ by Lemma 3 , it suffices to show that

$$
\left(D_{1},-D_{2}\right)_{2}=(-1)^{v_{11} v_{22}} \text {. }
$$

If $D_{1} \neq 0$, then (2.3) follows from Lemma 4 . If $D_{1}=0$, that is, $\boldsymbol{v}_{11}=0$, we have to show that $C_{2}(k)=1$. However, since $C_{2}(k)$ depends only on the $R$-equivalent class [2], to calculate $C_{2}(k)$ we may use $R M R^{\prime}$ $=\left(a_{i j}\right)$ instead of $M=V_{2}+V_{2}^{\prime}$ for some integral unimodular matrix $R$. $R$ may be chosen so that $a_{11} \neq 0$ and $a_{22}=0$. Then $C_{2}(k)=\left(a_{11}, a_{12}^{2}\right)_{2}$ $=1$.

Now we suppose that the theorem is true for $n<h$, and proceed to prove it for $n=h$.

Consider the surface $S^{\prime}$ obtained from $S$ by removing the last two bands $B_{2 h-1}$ and $B_{2 h}$. The genus of $S^{\prime}$ is $h-1$ and the boundary of $S^{\prime}$ is a new knot $k^{\prime}$. Moreover, $S^{\prime}$ is a Seifert surface of $k^{\prime}$ and $V_{2 h-2}$ is the Seifert matrix associated to $S^{\prime}$. Thus, by the induction assumption, we see that in order to prove the theorem it is sufficient to show that

$$
(-1)^{\beta+\beta^{\prime}}\left(-1,-D_{2 h}\right)_{2}\left(D_{2 h-2},-D_{2 h-1}\right)_{2}\left(D_{2 h-1},-D_{2 h}\right)_{2}\left(-1,-D_{2 h-2}\right)_{2}
$$

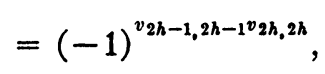

where $\beta^{\prime}=[(h-1) / 2]+h$.

Since $\beta+\beta^{\prime} \equiv h(\bmod 2)$ and

$$
\left(-1,-D_{2 h}\right)_{2}\left(-1,-D_{2 h-2}\right)_{2}=(-1)^{h+1}(-1)^{h}=-1
$$

by Lemma 3 , (2.4) reduces to

$$
(-1)^{h+1}\left(D_{2 h-2},-D_{2 h-1}\right)_{2}\left(D_{2 h-1},-D_{2 h}\right)_{2}=(-1)^{v 2 h-1,2 h-1^{02 h_{2} 2 h}} \text {. }
$$

If $D_{2 h-1}=0$, then $v_{2 h-1,2 h-1} v_{2 h, 2 h}$ must, by Lemma 1 , be an even number. The left-hand side of $(2.5)$ is, by our convention,

$$
(-1)^{h+1}\left(D_{2 h-2},-1\right)_{2}\left(1,-D_{2 h}\right)_{2}=(-1)^{h+1}(-1)^{h-1}=1 \text {. }
$$

Suppose $D_{2 h-1} \neq 0$. Then, since

$$
\begin{aligned}
\left(D_{2 h-2},-D_{2 h-1}\right)_{2} & =\left(D_{2 h-2},-1\right)_{2}\left(D_{2 h-2}, D_{2 h-1}\right)_{2} \\
& =(-1)^{h-1}\left(D_{2 h-2}, D_{2 h-1}\right)_{2},
\end{aligned}
$$

(2.5) reduces to

$$
\left(D_{2 h-1},-D_{2 h-2} D_{2 h}\right)_{2}=(-1)^{v_{2 h-1,2 h-1} v_{2 h, 2 h}} .=\text { 'ing }
$$

This follows from Lemma 4, however. Thus the proof of Theorem 1 is complete. 
Since Theorem 2 can be proved easily by induction on $h$, the details will be omitted.

\section{REFERENCES}

1. B. W. Jones, The arithmetic theory of quadratic forms, The Carus Math. Monographs No. 10, Math. Assoc. of America, Buffalo, N. Y., 1950.

2. K. Murasugi, On the Minkowski unit of slice links, Trans. Amer. Math. Soc. 114 (1965), 377-383.

3. R. A. Robertello, An invariant of knot cobordism, Comm. Pure Appl. Math. 18 (1965), 543-555.

4. H. Seifert, Über das Geschlecht von Knoten, Math. Ann. 110 (1934), 571-592.

UNIVERSITY OF TORONTO 